\title{
Der lange Weg der ASV in die Versorgungspraxis
}

Hält der G-BA, was der neue $\S 116 \mathrm{~b}$ SGB V verspricht?

\author{
PROJEKTGRUPPE \\ „AMBULANTE \\ SPEZIALFACHÄRZTLICHE \\ VERSORGUNG"IM \\ BUNDESVERBAND \\ MANAGED CARE E. V.
}

Autoren des Beitrages:

Roger Jaeckel, Leiter Gesundheitspolitik, GlaxoSmithKline (Leiter der Projektgruppe)

Sabine Barz, Referentin, Bundesverband Managed Care e. V.

Kristina Beyer, Healthcare Manager, Market Access, MSD Sharp \& Dohme GmbH

Dr. Christoph Bischoff-

Everding, HGC GesundheitsConsult $\mathrm{GmbH}$

Dr. Michaela Flug, Senior Health Care Manager Market Access \& Health Policy, Merck Serono GmbH

Sonja Froschauer,

Libertamed GmbH

Henning Kalk, BARMER GEK

Dr. Albrecht Kloepfer, Büro für gesundheitspolitische Kommunikation

Karl-Eberhard Schneider, CFL GmbH

Christina Unkenholz, KKH Kaufmännische Krankenkasse

\begin{abstract}
Mit der Neufassung des $\S 116 \mathrm{~b}$ SGB V hat die Politik Anfang 2012 den dritten Anlauf genommen, die Sektorengrenzen im Bereich der spezialfachärztlichen Versorgung nachhaltig zu überwinden. Ob dies auch in der Praxis gelingt, hängt in erheblichem Maß von der Konsensfindung des Gemeinsamen Bundesausschusses statt, der mit der Ausgestaltung der Details beauftragt wurde und im März 2013 eine erste Richtlinie zur ASV beschlossen hat. Die Projektgruppe „Ambulante spezialfachärztliche Versorgung“ des Bundesverbandes Managed Care e. V. (BMC) unternimmt im vorliegenden Beitrag einen Reality Check im Hinblick auf die Umsetzbarkeit der Vorgaben.
\end{abstract}

\section{Einführung}

Die Herausforderungen, vor denen das deutsche Gesundheitswesen aktuell steht, sind hinreichend bekannt: Die Bewältigung des demografischen Wandels und die schnelle Zunahme an chronisch kranken und multimorbiden Patienten stellen die kritischen Parameter dar, an denen die Zukunftsfähigkeit des Systems gemessen werden muss. Diese Entwicklungen erfordern nicht nur die Sicherstellung einer flächendeckenden, wohnortnahen Versorgung, sondern auch eine stärkere interdisziplinäre Zusammenarbeit zwischen den beteiligten medizinischen Disziplinen sowie zwischen ambulantem und stationärem Sektor.

Doch gerade an effizienten, harmonischen Kooperationsformen hapert es bislang. Verschiedene Vergütungssysteme, getrennte Budgets, unabhängige Bedarfsplanungen sowie eigene Entscheidungs- und Investitionsstrukturen führen dazu, dass die Mauer zwischen den Leistungssektoren nur schwer zu durch- brechen ist (vgl. Amelung/Wolf 2013, S. 114). Einerseits geht dieses zweigleisige System mit erheblichen Ineffizienzen einher, andererseits wird die starre Sektorentrennung mit dem raschen medizinischtechnologischen Fortschritt zunehmend hinfällig (vgl. Jaeckel 2012a, S. 19).

Der Gesetzgeber hat in den vergangenen zehn Jahren verschiedene Möglichkeiten geschaffen, um eine bessere Verzahnung zwischen den Leistungserbringern sowie zwischen den Sektoren zu initiieren (s. Abb. 1). Der große Durchbruch infolge dieser Reformen ist bisher jedoch ausgeblieben. Noch immer belaufen sich die Ausgaben für Integrierte Versorgung an den GKV-Gesamtausgaben auf weniger als ein Prozent (vgl. SVR 2012, S. 349).

Mit den Neuregelungen zum \116b SGB V im Rahmen des GKVVersorgungsstrukturgesetzes nimmt die Politik einen weiteren Anlauf, diese nach wie vor ungelöste Schnittstellenproblematik anzugehen (vgl. Jaeckel 2012b, S. 117). Die ambulante spezialfachärztliche Versorgung zielt darauf $a b$, eine 
Abbildung 1: Reformansätze zu § 116b SGB V zur Überwindung der Sektorengrenzen

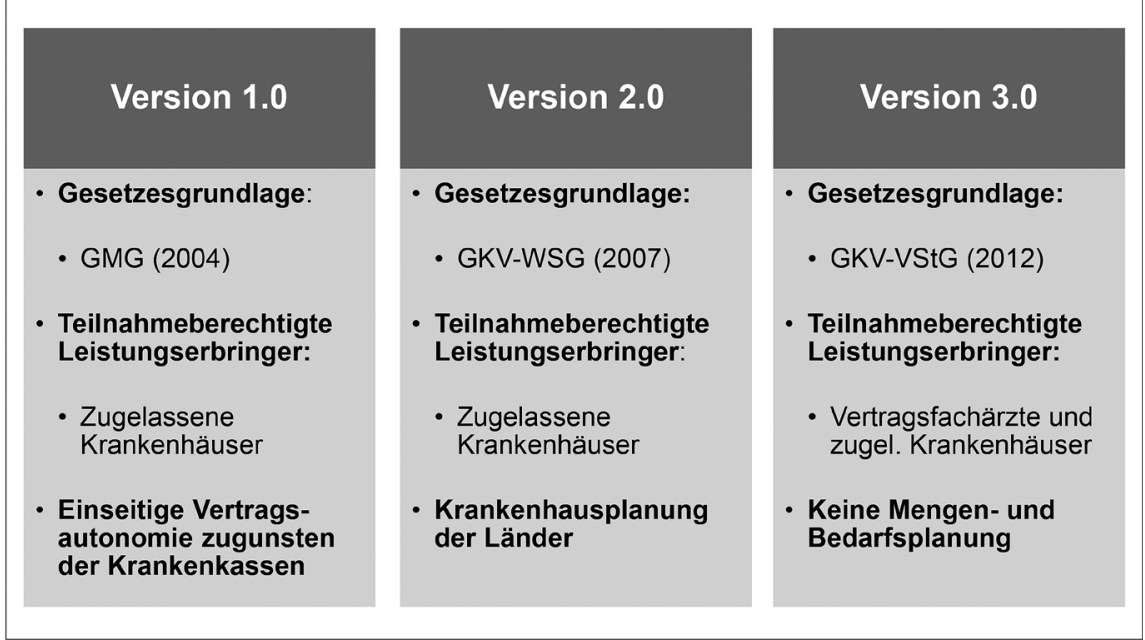

Quelle: GSK, Abt. Gesundheitspolitik 2013

\section{In welchem Szenario ist die ASV angesiedelt?}

Die ASV umfasst die ambulante Diagnostik und Behandlung von schweren Verlaufsformen von Erkrankungen mit besonderen Krankheitsverläufen, seltenen Erkrankungen und Erkrankungszuständen mit entsprechend geringen Fallzahlen sowie bestimmte hochspezialisierte Leistungen. Das Gesetz gibt hierbei bereits konkrete Krankheiten vor, jedoch ist der Katalog nicht abschließend. Auf Antrag kann der G-BA geeignete weitere Krankheitsbilder in die ASV aufnehmen. Dies wird laut Beschluss des G-BA jedoch erst dann der Fall sein, wenn die bislang vorgesehenen Erkrankungen bearbeitet wurden.

Im ersten Schritt hat der G-BA eine Priorisierung vorgenommen: Bei den schweren Verlaufsformen von Erkrankungen mit besonderen Krankheitsverläufen geht es zunächst um gastrointestinale Tumoren/Tumoren der Bauchhöhle, gynäkologische Tumoren, rheumatologische Erkrankungen sowie Herzinsuffizienz (NYHA 3 u. 4). Bei den seltenen Erkrankungen beschränkt sich die ASV vorerst auf Tuberkulose, Marfan-Syndrom, pulmonale Hypertonie, Mukoviszidose und primär sklerosierende Cholangitis. Zur Festlegung der „schweren Verlaufsform“ von Erkrankungen mit besonderen Krankheitsverläufen sollen nach ICD-10 verschlüsselte Diagnosen herangezogen werden - ggf. in Verbindung mit weiteren überprüfbabedarfsplanerische Maßnahmen noch leistungsbezogene Ausgabenbegrenzungen vorgesehen. Letzteres gilt auch für den Bereich der verordneten Leistungen, insbesondere Arzneimittel sowie Heilund Hilfsmittel.

Würde es mit diesem Ansatz gelingen, alte Versorgungsstrukturen aufzubrechen, ließe sich das Modell möglicherweise auch auf andere Versorgungsbereiche übertragen. Doch wie attraktiv die ASV letztlich für Krankenhäuser und Vertragsärzte sein wird, hängt in erheblichem Maße von der Konsensfindung des Gemeinsamen Bundesausschusses (G-BA) ab. Er wurde vom Gesetzgeber mit der Ausgestaltung der Details beauftragt und wird somit großen Einfluss auf die praktische Umsetzung der ASV haben (vgl. hierzu auch Munte 2013, S. 3 ff.).

Eine erste Richtlinie zum Geltungsbereich, zu den berechtigten Leistungser-

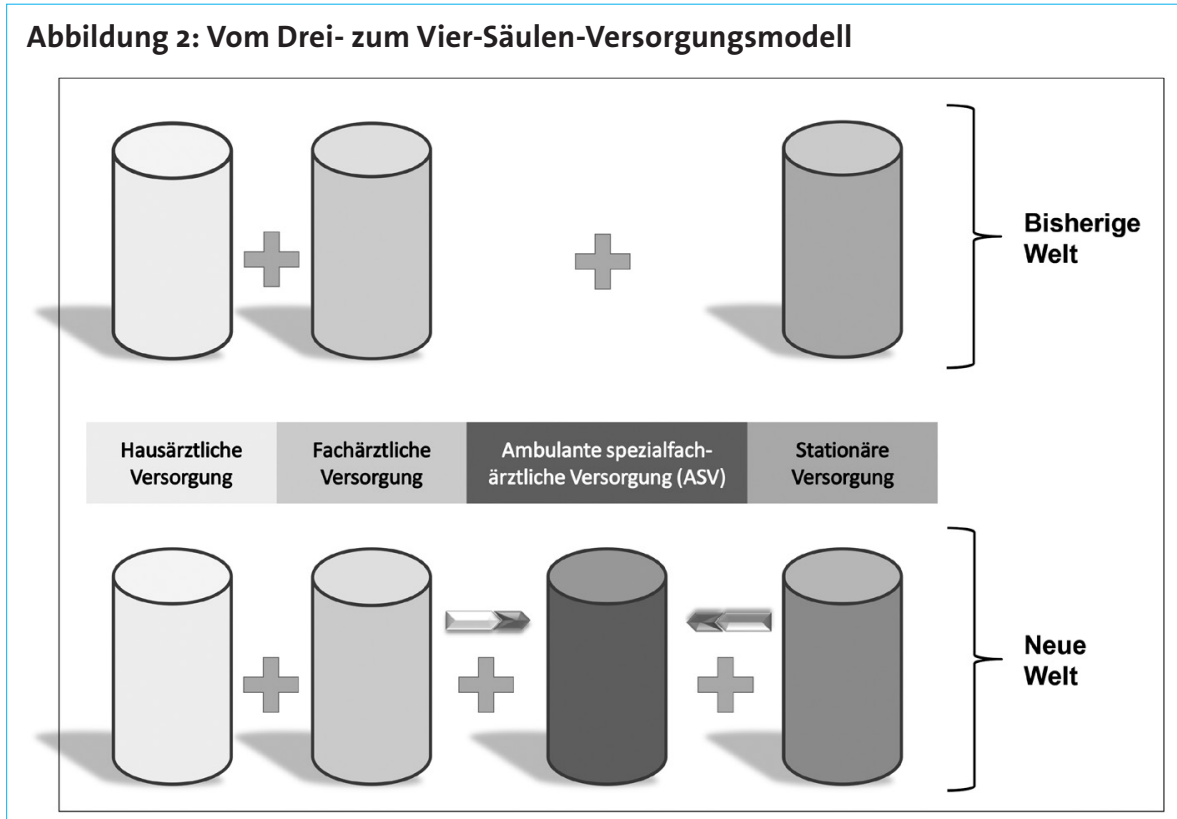

Quelle: Jaeckel 2012C, S. 3 
ren Kriterien wie indikationsspezifischen Klassifikationssystemen.

An der ASV können grundsätzlich Vertragsärzte und Medizinische Versorgungszentren (MVZ) sowie zugelassene Krankenhäuser ( $\mathbb{S} 108$ SGB V) teilnehmen. Der Teilnahmewunsch muss bei den in den KV-Regionen neu zu bildenden Erweiterten Landesausschüssen unter Nachweis sämtlicher Voraussetzungen und Anforderungen angezeigt werden. Der Erweiterte Landesausschuss prüft die Teilnahmeanzeige.

Die Vergütung erfolgt zunächst nach EBM, später sollen ambulante Fallpauschalen zugrunde gelegt werden.

\section{Der hohe Stellenwert der Kooperation ist richtungsweisend}

Das Herzstück der ASV besteht in der verpflichtenden Kooperation zwischen den Leistungserbringern. Die Teamzusammensetzung (s. Abb. 3) wird in den indikationsspezifischen Anlagen durch die Festlegung der geforderten Fachgruppen vorgegeben. Ein Team besteht grundsätzlich aus Teamleitung und Kernteam, wobei alle Mitglieder, auch im Krankenhaus tätige Ärzte, namentlich benannt werden müssen. Zusätzlich können weitere Fachärzte hinzugezogen werden, wenn dies aus medizinischen Gründen erforderlich ist. Hier reicht eine institutionelle Nennung aus.

Der hohe Stellenwert, den diese Kooperationsvorgaben in der ASV-Richtlinie (ASV-RL) einnehmen, ist richtungsweisend. Insbesondere ermöglicht die ASV auch Vertragsärzten außerhalb großer fachübergreifender Gemeinschaftspraxen und MVZ einen Zugang zu dieser neuen Versorgungsebene. Überdies können Krankenhäuser, die den geforderten Leistungsumfang nicht selbst abdecken, die fehlenden Anforderungen über eine Kooperation mit einem niedergelassenen Facharzt komplettieren.

$\mathrm{Zu}$ begrüßen ist, dass der G-BA bei den Entfernungsvorgaben Entscheidungsspielräume gelassen hat. Diese sollten durch die Erweiterten Landesausschüsse unter Berücksichtigung der regionalen Besonderheiten genutzt werden.

Für onkologische Erkrankungen besteht in der ASV zusätzlich zu den allgemeinen Kooperationserfordernissen die Verpflichtung zur sektorenübergreifenden Kooperation (sog. ASVKooperation). Es muss eine vertraglich
Abbildung 3: Zusammensetzung der interdisziplinären Teams

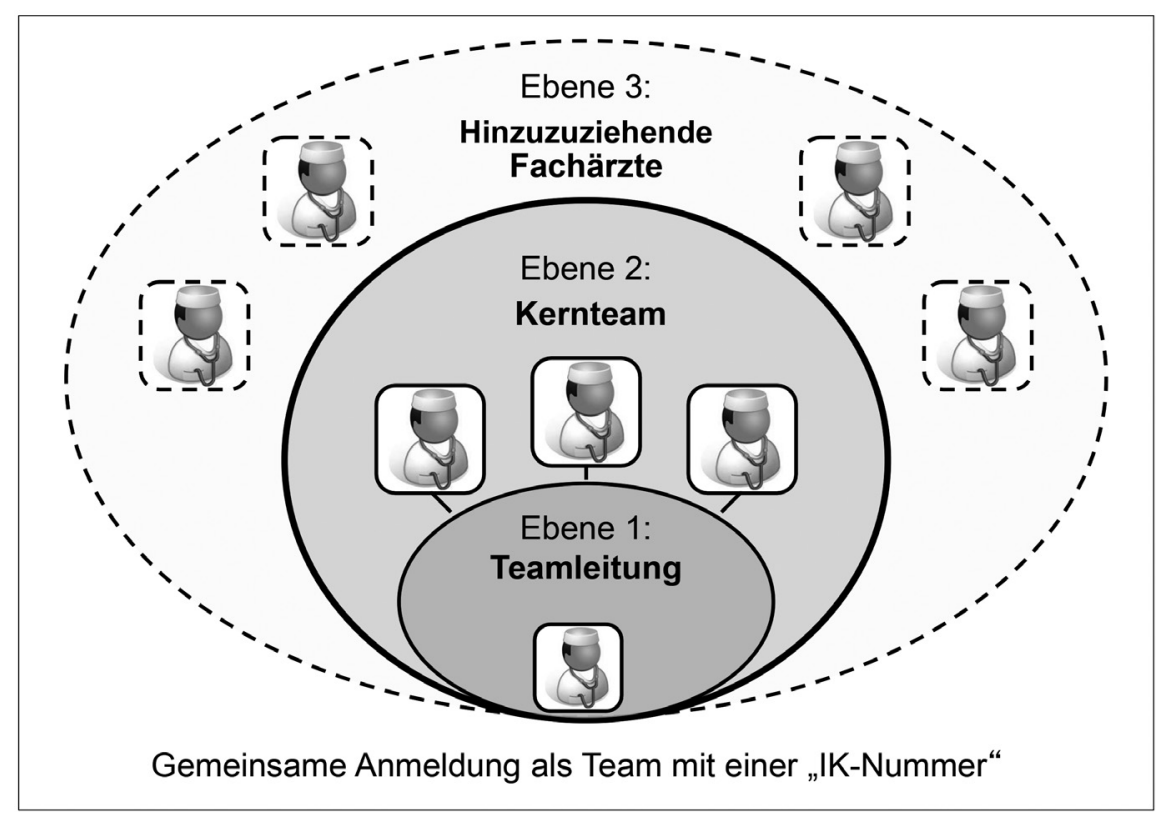

Quelle: GSK, Abt. Gesundheitspolitik 2013

vereinbarte Zusammenarbeit zwischen den Leistungserbringern sowohl aus dem ambulanten als auch aus dem stationären Bereich geben, die u. a. die Absprache über Vorgehensweisen sowie mindestens zweimal jährlich stattfindende qualitätsorientierte Konferenzen vorsieht.

Die Vorgaben zur ASV-Kooperation orientieren sich stark an bereits etablierten Tumorkonferenzen. Dies ist sinnvoll, da so auf vorhandenen Strukturen aufgebaut werden kann. Um Pseudokooperationen zu vermeiden, fehlen zurzeit jedoch noch weitere Vorgaben für diese ASV-Kooperationen. So geht aus der ASV-Richtlinie u. a. nicht hervor, welche Voraussetzungen die Kooperationspartner erfüllen müssen (z. B. Nachweis einer bestimmten ärztlichen Fachgruppenzugehörigkeit). Dies erscheint vor allem vor dem Hintergrund der Ausnahmeregelung relevant, nach der eine ASV-Teilnahme in der Onkologie auch ohne ASV-Kooperation möglich ist, sofern kein Kooperationspartner gefunden werden konnte.

Ein anderer Aspekt betrifft das Verhältnis der beiden Kooperationsebenen zueinander. So ist nicht eindeutig beschrieben, ob die Vertragspartner der sektorenübergreifenden Kooperation gleichzeitig Mitglieder des interdisziplinären Teams sein können.

Eine Klärung dieser Punkte ist entscheidend für die regionale Umsetzung, da sie letztlich das Angebot an potenziellen Kooperationspartnern bestimmen.
Hier sollte der G-BA im Konkretisierungsteil weitere Details regeln.

\section{Wie kommt der Patient in die neue Versorgungsstruktur?}

Um Patienten möglichst koordiniert in die ASV überführen zu können, ist laut G-BA-Richtlinie im Bereich der schweren Verlaufsformen grundsätzlich die Überweisung durch Vertragsärzte erforderlich. Bei seltenen Erkrankungen und hochspezialisierten Leistungen kann der G-BA dies ebenfalls vorgeben. Patienten, die zuvor im Krankenhaus stationär behandelt wurden, benötigen keine Überweisung.

Zwischen den Mitgliedern des Kernteams sind für ASV-Patienten ebenfalls keine Überweisungen nötig. Wenn weitere Fachärzte hinzugezogen werden, erbringen diese ihre Leistungen entsprechend dem jeweiligen Behandlungsumfang auf Überweisung. $\mathrm{Ob}$ und in welchem Umfang eine Überprüfung der Indikation vorzunehmen ist, wird im Zusammenhang mit der Erstellung der indikationsspezifischen Anlagen abschließend geregelt.

Die gesicherte Diagnose als Überweisungs- bzw. Zuweisungsvoraussetzung im Bereich der schweren Verlaufsformen ist aus Sicht des BMC sinnvoll. Für die Zuweisungen aus dem stationären Bereich in die ASV sollte jedoch ein konkreter ASV-Fallbezug nachgewiesen werden, 
um eine zielgerichtete Patientenversorgung sicherzustellen.

Positiv ist auch die regelmäßige Überprüfung des Fortbestehens einer ASVIndikation zu sehen. Die Vorgaben der ärztlichen Prüfung, ob die ASV-Indikation noch gegeben ist, muss allerdings unbürokratisch und dennoch einheitlich erfolgen. In Bezug auf seltene Erkrankungen scheint die Verdachtsdiagnose als Überweisungskriterium zunächst ausreichend.

Nach der Überweisung bzw. Zuweisung in die ASV erhalten die Patienten allgemeine Informationen über diese neue Versorgungsform sowie über die Zusammensetzung und das Leistungsspektrum des behandelnden interdisziplinären Teams. Nach Abschluss ihrer ASVBehandlung bekommen sie einen Bericht über die Behandlungsergebnisse und das weitere Vorgehen. Wird die Behandlung außerhalb der ASV durch Ärzte fortgesetzt, die nicht zum Kernteam gehören, werden ein verständlicher Entlass-/ Überleitungsbrief sowie eine Anleitung zur Fortsetzung der Arzneimitteltherapie bereitgestellt. Der in die ASV überweisende Facharzt wird zudem über das Ende der ASV-Behandlung in Kenntnis gesetzt. Sämtliche Informationen, die der Patient während seiner Behandlung in der ASV erhält, sind durch den ASVteilnehmenden Arzt zu dokumentieren.

Transparente Patienteninformationen können dazu beitragen, die Patienten schneller an die neue Versorgungsstruktur heranzuführen und Vertrauen in diese neue Versorgungsform zu schaffen. Eine Einbindung der Patientenvertretungen bei der Entwicklung von Vorgaben erscheint sinnvoll.

\section{Kontinuität der ASV- Leistungserbringung}

Ist der Patient erst einmal in die ASV aufgenommen, muss eine kontinuierliche Behandlung gewährleistet sein, zumal es sich bei den betreffenden Indikationen um langwierige, teilweise chronische Erkrankungen handelt. Da das Kernteam stets aus namentlich benannten Ärzten besteht, müssen auch Vertretung und Nachbesetzung personenbezogen geregelt sein. Der G-BA hat in seiner Richtlinie hierzu umfassende Regelungen formuliert. Demnach darf die Vertretung der Mitglieder nur durch Fachärzte erfolgen, die die Richtlinien-Anforderungen des G-BA nachweislich erfüllen (z. B. fachliche Qua- lifikation, organisatorische Einbindung). Bei einer Vertretungsdauer von mehr als einer Woche ist diese dem Erweiterten Landesausschuss, den Landesverbänden der Krankenkassen und dem Verband der Ersatzkassen, der Kassenärztlichen Vereinigung (KV) sowie der Landeskrankenhausgesellschaft zu melden.

Gleichwohl bleiben aus BMC-Sicht folgende Sachverhalte klärungsbedürftig:

- Wie verhält es sich mit der Behandlung von ASV-Patienten, wenn dem gesamten ASV-Team die Berechtigung entzogen wird (z. B. wenn nach Ausscheiden eines Mitglieds keine Nachbesetzung erfolgen kann)?

- In welcher Form hat die Meldung der Vertretung bzw. Nachbesetzung zu erfolgen?

- Bedarf die Inanspruchnahme einer Vertretung grundsätzlich einer dezidierten Begründung (z. B. Urlaub, Krankheit, Fortbildung)?

Insgesamt führen die Vertretungs- und Nachbesetzungsregelungen sowohl bei den Leistungserbringern als auch bei den Erweiterten Landesausschüssen zu einem erheblichen bürokratischen Aufwand. Aus Sicht des BMC dürfen die Vorgaben nicht zu einer Verkomplizierung der Patientenversorgung im Bereich der ASV führen, sondern müssen praxistauglich und möglichst bürokratiearm gestaltet werden. Insofern empfiehlt der BMC dem G-BA, diesen Regelungsabschnitt nochmals zu überprüfen. Wenn es beispielsweise durch

\section{Die Nachbesetzungs- und Vertretungsregelungen sind mit großem bürokratischen Aufwand verbunden.}

das Ausscheiden eines Teammitglieds zu einer Status-Aberkennung des gesamten ASV-Teams kommt, weil innerhalb von sechs Monaten keine Nachbesetzung erfolgen konnte, darf sich dies nicht nachteilig auf die Patientenversorgung auswirken.

\section{Wie wird die Qualität der ASV sichergestellt?}

Angesichts der Krankheitsbilder in der ASV sollte die Qualitätssicherung höchsten Standards genügen. Die in der $\mathbb{1 1 6 b -}$
Richtlinie a. F. bereits bestehenden Vorgaben zur Qualitätssicherung - soweit für die ASV-Leistungen relevant - finden auch im neuen Versorgungsbereich Anwendung. Parallel hierzu wird der Geltungsbereich der Qualitätssicherungsvereinbarungen nach $\$ 135$ Abs. 2 SGB V für die vertragsärztliche Versorgung im Rahmen der ASV nunmehr auch auf den stationären Bereich übertragen. Die Überprüfung der Qualität obliegt dabei den Krankenkassen.

Neben Kriterien zur Strukturqualität der teilnehmenden Leistungserbringer sollten aus Sicht des BMC insbesondere auch Maßnahmen zur Indikationssicherung mit aufgenommen werden. Die ASV bietet außerdem die Chance, Parameter zur Messung der Ergebnisqualität zu erheben, um beispielsweise den ASVTeams Transparenz über die Qualität ihrer Arbeit zu ermöglichen.

Offen ist jedoch die Frage, wie die Durchführung der Qualitätsprüfungen organisiert werden soll. Dies ist mit signifikantem Aufwand verbunden - so sehen beispielsweise viele Qualitätssicherungsvereinbarungen laufende Stichprobenprüfungen vor, die dann auch bei Krankenhäusern durchgeführt werden müssten. Im Interesse einheitlicher Standards und zur Vermeidung von Wettbewerbsnachteilen einzelner Leistungserbringer sollte eine kassen- und ggf. auch länderübergreifende Lösung angestrebt werden.

Ein wichtiges Element der Qualitätssicherung besteht in der sektorenübergreifend einheitlichen Leistungsdokumentation, wie der G-BA sie in seiner Richtlinie für alle ASV-Leistungserbringer vorsieht. Für die Leistungsbewertung wird aber zusätzlich die Verfügbarkeit einer entsprechenden Datenbasis (Dokumentation) relevant sein. Die Forderung, dabei vermehrt auf Routinedaten (meist Abrechnungsdaten) zurückzugreifen, erscheint zwar sinnvoll, dürfte aber schwer umsetzbar sein. Da in der ASV eine bilaterale Abrechnung der Leistungserbringer mit den einzelnen Krankenkassen möglich ist, fehlt ein übergreifender zentraler Datenbestand. Auch sind Abrechnungsdaten oft nicht ausreichend, um die Qualität bewerten zu können, sodass ergänzende Dokumentationen notwendig sind. Welche Inhalte hier relevant sind, sollte aus Sicht des BMC in den konkretisierenden Anlagen zu den einzelnen Indikationen geregelt werden. 
Sinnvoll erscheint hier die Zusammenführung aller Daten bei einer zentralen Vertrauensstelle, in der Dokumentationen und Evaluationen auf EDV-Basis gesammelt werden. Die so entstehende Datenbank könnte zugleich die Basis für eine gemeinsame elektronische Fallakte bilden. Zudem ließe sie sich im Rahmen der Versorgungsforschung beispielsweise zu seltenen Erkrankungen nutzen.

Ein weiteres Instrument zur Qualitätssicherung kann die Mindestmengenregelung darstellen. Hierzu hat der G-BA bisher keinen allgemein gültigen Beschluss gefasst, sondern lediglich darauf verwiesen, dass eventuelle Mindestmengen in den indikationsspezifischen Anlagen geregelt werden. Aus Sicht des BMC sollten Mindestmengen nur dort gefordert sein, wo ein evidenzbasierter Zusammenhang zwischen Menge und Behandlungsqualität besteht. Jedoch muss dabei gewährleistet sein, dass auch

\section{Bei der Evaluation sollte ein Fokus auf den Mehrwert gegenüber der bestehenden fachärztlichen Versorgung gelegt werden.}

qualitätsorientierte regionale Strukturen die Chance erhalten, sich im Wettbewerb zu etablierten Strukturen zu entwickeln.

Nach den gesetzlichen Vorgaben ist nach fünf Jahren ein Evaluationsbericht vorzulegen, der insbesondere den Stand der Versorgungsstruktur, die Qualität sowie die Abrechnung der Leistungen auch im Hinblick auf die Entwicklung in anderen Versorgungsbereichen beinhaltet.

$\mathrm{Da}$ die ASV flächendeckend in Deutschland eingeführt wird, vergibt sich das Versorgungssystem - ähnlich wie bei der Einführung der DiseaseManagement-Programme (DMP) vor über zehn Jahren - die Chance, durch eine Nicht-ASV-Gruppe die gesundheitsökonomischen und qualitativen Effekte auf die Versorgung von seltenen Erkrankungen und Erkrankungen mit schweren Krankheitsverläufen wissenschaftlich zu messen und auszuwerten. Vergleiche zwischen Prozess- und vor allem Ergebnisqualität von ASV einerseits und fortgeführter Sektorentrennung im Rahmen der Regelversorgung andererseits werden nicht darstellbar sein. Es stellt sich daher die Frage, ob aus der Versorgungspraxis heraus und ohne zeitnahe wissenschaftliche Begleitforschung die vorgeschriebene Evaluation den Ansprüchen einer aussagekräftigen Bewertung überhaupt gerecht werden kann. In jedem Fall sollte bei der Evaluation ein Fokus auf dem Mehrwert gegenüber der bestehenden fachärztlichen Versorgung liegen. Nur dadurch lässt sich der hohe Aufwand, der bei allen Beteiligten mit dem Auf- und Ausbau eines neuen Versorgungsangebotes einhergeht, fachlich und politisch begründen.

\section{Innovationsorientierung versus Qualitätsanspruch: Verbotsvorbehalt}

Das grundsätzlich nur für den stationären Bereich geltende Prinzip des Verbotsvorbehalts ( $\mathbb{S} 137 \mathrm{c}$ SGB V) kann nunmehr auch für die ASV angewandt werden. Dieses Prinzip besagt, dass neue Untersuchungs- und Behandlungsmethoden (NUB) prinzipiell ohne vorherige Prüfung durch den G-BA zulasten der GKV erbracht werden können, solange der G-BA nicht ausdrücklich etwas anderes beschließt.

Mit der jetzt beschlossenen G-BA-Richtlinie erfährt diese Regelung jedoch eine Einschränkung. Es werden nur die für die ASV geeigneten NUB in den noch zu beschließenden indikationsspezifischen Anlagen aufgeführt. Damit praktiziert die Gemeinsame Selbstverwaltung eine Leistungssteuerung, für die allerdings klare Prozesse definiert werden müssten. Wichtig wäre darüber hinaus, spezielle Qualitätskriterien zu definieren, die die Ärzte und Krankenhäuser erfüllen müssen, um NUB zu erbringen. Hier muss gewährleistet sein, dass NUB nur an entsprechend qualifizierten Zentren erbracht werden.

Um die vom Gesetzgeber eindeutig vorgegebene Prämisse nicht zu konterkarieren, schlägt der BMC folgende alternative Ergänzungsregelungen vor:

- Alternative 1: Vorgabe eines strukturierten Entscheidungsprozesses mit klaren Entscheidungskriterien und zeitlichen Fristen für die Einführung von NUB

- Alternative 2: Anwendung einer Bewertungsmethodik analog dem INEKVerfahren

\section{Bewertung weiterer wichtiger Aspekte zur Neuregelung der ASV}

Weitere wichtige Aspekte, die im Rahmen der Neufassung des $\mathbb{S} 116 \mathrm{~b}$ SGB V geregelt wurden, jedoch nicht im $\mathrm{Zu}$ sammenhang mit der G-BA-Richtlinie stehen, sind die Abrechnung der ASVLeistungen und die Einrichtung der Erweiterten Landesausschüsse.

\subsection{Abrechnung der ASV-Leistungen}

Der G-BA legt für jedes Krankheitsbild in der ASV den Behandlungsumfang fest. Das bedeutet, dass er diejenigen ambulanten Leistungen auf Ebene einzelner Gebührenordnungspositionen abschließend definiert, die im Rahmen der ASV für einen Patienten mit dem jeweiligen Krankheitsbild erbracht und abgerechnet werden können.

In der Anfangszeit werden ASV-Leistungen sowohl durch Krankenhäuser als auch durch Vertragsärzte, die an der ASV teilnehmen, nach dem Einheitlichen Bewertungsmaßstab (EBM) abgerechnet. Die Vergütung erfolgt extrabudgetär. Krankenhäuser müssen jedoch einen fünf prozentigen Abschlag von der Vergütung hinnehmen, um die Investitionsförderung im Rahmen der dualen Krankenhausfinanzierung auszugleichen. In einer Übergangsphase soll vom Erweiterten Bewertungsausschuss eine eigene Gebührenordnung für die ASV entwickelt werden. Letztlich soll die ASV-Vergütung gemeinsam vom GKV-Spitzenverband, der Deutschen Krankenhausgesellschaft (DKG) und der Kassenärztlichen Bundesvereinigung (KBV) in ein eigens dafür entwickeltes diagnoseabhängiges Gebührenordnungssystem überführt werden.

Der BMC begrüßt die vom G-BA vorgenommene Festlegung des Behandlungsumfangs und der somit zulasten der GKV abrechenbaren Leistungen anhand des EBM. Diese Vorgehensweise stellt eine praktikable Zwischenlösung zur Umsetzung der ASV dar. Zudem entstehen dadurch keine unnötigen Mehrkosten. Um Doppelabrechnungen zu vermeiden bzw. identifizieren zu können, ist es allerdings wichtig, eindeutige EBM-Ziffern für die ASV festzulegen. Bei den Krankenkassen werden anhand der Daten entsprechende Plausibilitätsprüfungen etabliert, was aber mit zusätzlichem Verwaltungsaufwand verbunden ist.

Eine Abrechnungsprüfung insbesondere im Hinblick auf Doppelabrechnun- 
gen durch die Kassenärztlichen Vereinigungen (KVen) ist hingegen nur eingeschränkt möglich, da dort aufgrund der Möglichkeit der direkten Abrechnung mit den Kassen nicht mehr alle Daten zusammenlaufen. Hierdurch entfällt ein etabliertes Prüfverfahren. Im vertragsärztlichen Bereich ist zudem eine Bereinigung vorgesehen, wobei der damit verbundene Verwaltungsaufwand bei den Krankenkassen und den KVen nicht $\mathrm{zu}$ unterschätzen ist. Wichtig ist hier, diese mit möglichst geringem Verwaltungsaufwand und klaren Regelungen für die Beteiligten auszugestalten.

Kritisch stellt sich aus Sicht des BMC auch die Möglichkeit mehrerer Abrechnungswege und unterschiedlicher Datenformate dar (Ambo-Datensatz nach $\mathbb{S} 301$ SGB V und Daten nach \295 Abs. 1b SGB V). Bei der Prüfung der Abrechnungsdaten müssen dann ggf. Daten aus verschiedenen Abrechnungswegen und regional bedingt verschiedenen Formaten in ein einheitliches Format gebracht werden.

Die künftig auch für den vertragsärztlichen Bereich vorgesehene direkte Leistungsabrechnung kann für Ärzte und Krankenkassen aufwendig und zeitraubend sein. Die Abrechnung über einen Dienstleister ist ebenso mit Kosten verbunden. Für die Krankenhäuser ist die Direktabrechnung mit den Krankenkassen sowie die Verwendung des Ambo-Datensatzes ein etabliertes Verfahren, über das bereits jetzt ambulante Krankenhausleistungen abgerechnet werden. Sollte dieses Datenübermittlungsverfahren auch für den niedergelassenen Bereich umgesetzt werden, ist noch fraglich, ob eine reibungslose Kompatibilität mit der Praxissoftware möglich sein wird. Unter wirtschaftlichen Aspekten ist es aus BMC-Sicht sinnvoll, kassenarten- und sektorenübergreifende Prüfungen einer einheitlichen Stelle zu übertragen, sofern dies unter datenschutzrechtlichen Gesichtspunkten möglich ist.

Für eine erfolgreiche Implementierung der ASV wird es entscheidend sein, dass Leistungserbringer die erbrachten Leistungen abrechnen und die Daten hierzu den Krankenkassen zur Verfügung stellen können. Zudem müssen die Krankenkassen diese Daten annehmen und verarbeiten können. Langfristig sollten bundesweit praktikable, einheitliche Standards auf eine gesetzliche Grundlage gestellt und somit ein einheitliches Abrechnungsverfahren etabliert werden.
Die Abrechnung der Vertretungsleistungen ist aktuell ungeklärt, insbesondere die Frage, über wen die Abrechnung erfolgt (ASV-Berechtigte oder Vertreter) und wie die Vertretungsleistung gekennzeichnet ist.

\subsection{Erweiterte Landesausschüsse}

Zur Umsetzung der ASV in die Versorgungspraxis hat der Gesetzgeber mit dem Erweiterten Landesausschuss auf regionaler Ebene ein entsprechendes Entscheidungsgremium geschaffen. Die wesentliche Aufgabe besteht darin, die Anforderungsvoraussetzungen der ASV-berechtigten Leistungserbringer zu überprüfen.

Für eine reibungslose Umsetzungspraxis der ASV wird es vor allem darauf ankommen, dass die Erweiterten Landesausschüsse zeitnah funktionsfähig und flächendeckend etabliert werden. Nach aktuellem Kenntnisstand sind diese Gremien jedoch noch nicht in allen KV-Bezirken konstituiert.

Derzeit gibt es für die operative Umsetzung der Qualifikationsprüfungen keine Empfehlungen, beispielsweise in Form einer vom G-BA definierten Verfahrensordnung für die Erweiterten Landesausschüsse. Damit besteht die Gefahr einer regional unterschiedlichen Prüfungs- und Auslegungspraxis. Eine solche regional unterschiedliche Umsetzung war bereits beim $\mathbb{S} 116$ b SGB V a. F. zu beobachten und könnte bei der ASV zu Wettbewerbsverzerrungen führen.

Zur Sicherstellung einer gleichwertigen Umsetzungspraxis in den neuen Landesgremien empfiehlt der BMC einen strukturierten Abstimmungsprozess der Beteiligten. Zur Gewährleistung einer gleichbleibenden Strukturqualität in der ASV sollte die Richtlinienkompetenz des G-BA in diesem Zusammenhang um Handlungsempfehlungen erweitert werden. Es sollten die Regelungspunkte unterschieden werden, die eine bundesweite Umsetzungspraxis benötigen bzw. einen regionalen Ermessensspielraum erfordern.

\section{Beachtung von Schnittstellen zu anderen Versorgungsbereichen}

Verschiedene Krankheitsbilder der ASV weisen unmittelbare Schnittstellen zu anderen Versorgungsbereichen auf, so z. B. im Bereich der Spezialisierten Ambulanten Palliativversorgung (SAPV), der Allgemeinen Ambulanten Palliativversorgung (AAPV) sowie von indikations- oder populationsbezogenen
Versorgungsverträgen $(\mathbb{S} \mathbb{S} 73 \mathrm{a}, \mathrm{b}, \mathrm{c}$, $\mathbb{S} \mathbb{S} 140 \mathrm{ff}$. SGB V etc.).

Offen ist, wie die Zusammenarbeit der Leistungserbringer an den Schnittstellen erfolgt und wie Leistungsüberschneidungen in der Praxis vermieden bzw. gelebt werden. Aus Patientensicht stellt sich die Frage, ob durch die Teilnahme an verschiedenen Verträgen die ASV-Behandlung grundsätzlich ausgeschlossen ist.

Die Festlegung der ASV-Leistungen muss unter Berücksichtigung der Versorgungsschnittstellen erfolgen, um Unsicherheiten zu vermeiden, d. h., die nach dem SGB V definierten Versorgungsbereiche müssen klar voneinander abgegrenzt werden. Zudem muss die Zusammenarbeit der Leistungserbringer unterschiedlicher Versorgungsbereiche (z. B. ASV und SAPV) geregelt werden. In keinem Fall dürfen vorhandene Schnittstellen zu Nachteilen für die Patienten führen. Auch dürfen sich aus der ASV keine Nachteile für die Umsetzung der SAPV ergeben. Wichtig ist darüber hinaus, dass die unterschiedlichen Versorgungsangebote den Patienten transparent vermittelt werden und dass sie auf ihr Recht hingewiesen werden, die Weiterbehandlung im Rahmen der SAPV in Anspruch nehmen zu können.

\section{Fazit: Wie praxistauglich ist das Verfahren zur Einführung neuer Versorgungsformen?}

Die eingangs erwähnten Ziele, die der Gesetzgeber mit den Neuregelungen zur ASV verfolgt, entsprechen grundsätzlich den Anforderungen an ein zukunftsfähiges Gesundheitssystem. Es stellt sich allerdings die Frage, ob das Verfahren, das gewählt wurde, um diese neue Versorgungsstruktur in die Praxis zu übertragen, sinnvoll, praktikabel und zielführend ist. Während die Politik die Rahmenbedingungen vorgibt, ist der G-BA damit beauftragt, dieses Gerüst mit Leben zu füllen. Bereits der Gesetzestext ist ein umfangreiches und komplexes sprachliches Gebilde, das ohne juristische Kompetenz kaum zu erfassen ist. Und es ist zu erwarten, dass die daraus abgeleiteten Richtlinien des G-BA den Umfang des Gesetzes am Ende um ein Vielfaches übersteigen werden.

Dabei sind viele Aspekte trotz umfangreicher Regelwerke bei Weitem nicht erschöpfend geklärt. So sollen qua Gesetz zwar beide Sektoren gleichberechtigten Zugang zur ASV erhalten. Die Vergütungs- 
Tabelle 1: Bewertung der ASV-Richtlinie und weiterer ASV-Bereiche aus Sicht des BMC

\begin{tabular}{|c|c|c|c|}
\hline Bereich & $\begin{array}{c}\square \\
\text { Positiv zu bewerten }\end{array}$ & $\stackrel{?}{\text { Klärungsbedarf }}$ & Empfehlungen \\
\hline Kooperation & $\begin{array}{l}\text { Hoher Stellenwert der Kooperation } \\
\text { Ansatz des gleichberechtigten Zugangs } \\
\text { von Krankenhäusern und Vertragsärzten } \\
\text { Orientierung an den Tumorkonferenzen } \\
\text { bei der sektorenübergreifenden ASV- } \\
\text { Kooperation im Bereich der Onkologie }\end{array}$ & $\begin{array}{l}\text { Welche Voraussetzungen (z. B. Nach- } \\
\text { weis Fachgruppenzugehörigkeit) müs- } \\
\text { sen die Kooperationspartner bei der sog. } \\
\text { sektorenübergreifenden Kooperation im } \\
\text { Bereich der Onkologie erfüllen? } \\
\text { Können die Vertragspartner der } \\
\text { sektorenübergreifenden Kooperation } \\
\text { gleichzeitig Mitglieder des interdiszipli- } \\
\text { nären Teams sein? }\end{array}$ & \\
\hline $\begin{array}{l}\text { Überweisung/ } \\
\text { Zuweisung }\end{array}$ & $\begin{array}{l}\text { Gesicherte Diagnose als Überweisungs- } \\
\text { bzw. Zuweisungsvoraussetzung im } \\
\text { Bereich der schweren Verlaufsformen } \\
\text { Vorgabe der regelmäßigen Überprüfung } \\
\text { des Fortbestehens einer ASV-Indikation }\end{array}$ & & $\begin{array}{l}\text { Nachweis des konkreten ASV-Fallbezugs } \\
\text { bei Zuweisungen aus dem stationären } \\
\text { Bereich } \\
\text { Unbürokratische, aber einheitliche } \\
\text { Vorgaben zur ärztlichen Überprüfung, } \\
\text { ob weiterhin eine ASV-Indikation } \\
\text { gegeben ist }\end{array}$ \\
\hline $\begin{array}{l}\text { Vertretung/ } \\
\text { Nachbesetzung }\end{array}$ & & $\begin{array}{l}\text { Wie kann die Versorgungskontinu- } \\
\text { ität sichergestellt werden, wenn dem } \\
\text { gesamten ASV-Team die Berechtigung } \\
\text { entzogen wird? } \\
\text { In welcher Form hat die Meldung der } \\
\text { Vertretung bzw. Nachbesetzung zu } \\
\text { erfolgen? } \\
\text { Bedarf die Inanspruchnahme einer Ver- } \\
\text { tretung grundsätzlich einer dezidierten } \\
\text { Begründung? }\end{array}$ & $\begin{array}{l}\text { Praxistaugliche, bürokratiearme Vertre- } \\
\text { tungs- und Nachbesetzungsregelungen }\end{array}$ \\
\hline $\begin{array}{l}\text { Qualitätssicherung/ } \\
\text { Evaluation }\end{array}$ & & $\begin{array}{l}\text { Wie wird die Durchführung von Quali- } \\
\text { tätsprüfungen organisiert? } \\
\text { Welche Datenbestände werden für } \\
\text { Qualitätsprüfungen herangezogen? }\end{array}$ & $\begin{array}{l}\text { Einführung von Maßnahmen zur Indika- } \\
\text { tionssicherung } \\
\text { Kassen- und ggf. länderübergreifende } \\
\text { Durchführung von Qualitätsprüfungen } \\
\text { Regelung in den konkretisierenden } \\
\text { Anlagen, welche Datenbestände zur } \\
\text { Qualitätssicherung herangezogen } \\
\text { werden dürfen } \\
\text { Zusammenführung aller Dokumen- } \\
\text { tationen und Abrechnungsdaten bei } \\
\text { zentraler Vertrauensstelle } \\
\text { Einführung von Mindestmengen nur bei } \\
\text { evidenzbasiertem Zusammenhang zwi- } \\
\text { schen Menge und Behandlungsqualität } \\
\text { Fokus auf Mehrwert gegenüber der } \\
\text { bestehenden fachärztlichen Versorgung } \\
\text { bei der Evaluierung }\end{array}$ \\
\hline NUB & & & $\begin{array}{l}\text { Klar definierte Qualitätskriterien für die } \\
\text { Berechtigung, im Rahmen der ASV NUB } \\
\text { zu erbringen } \\
\text { Ergänzungsregelung NUB: strukturierter } \\
\text { Entscheidungsprozess mit klaren Kriterien } \\
\text { und zeitlichen Fristen oder Bewertungs- } \\
\text { methodik nach dem INEK-Verfahren }\end{array}$ \\
\hline \multicolumn{4}{|c|}{ Außerhalb der Richtlinienkompetenz des G-BA } \\
\hline Abrechnung & $\begin{array}{l}\text { Vom G-BA vorgenommene Festlegung } \\
\text { des Behandlungsumfangs und somit } \\
\text { der abrechenbaren Leistungen anhand } \\
\text { des EBM als praktikable Zwischenlösung }\end{array}$ & $\begin{array}{l}\text { Wie bzw. über wen werden Vertretungs- } \\
\text { leistungen abgerechnet (ASV-Berechtig- } \\
\text { te oder Vertreter)? } \\
\text { Wie wird die Vertretungsleistung in der } \\
\text { Abrechnung gekennzeichnet? }\end{array}$ & $\begin{array}{l}\text { Festlegung eindeutiger EBM-Ziffern für } \\
\text { die ASV } \\
\text { Abrechnungsprüfung mit geringem } \\
\text { Verwaltungsaufwand } \\
\text { Einheitliches kassenarten- und } \\
\text { sektorenübergreifendes Abrech- } \\
\text { nungsverfahren }\end{array}$ \\
\hline $\begin{array}{l}\text { Erweiterte Landes- } \\
\text { ausschüsse }\end{array}$ & & $\begin{array}{l}\text { Wie kann verhindert werden, dass die } \\
\text { Qualifikationsprüfungen regional unter- } \\
\text { schiedlich erfolgen? }\end{array}$ & $\begin{array}{l}\text { Erweiterung der Richtlinienkompetenz } \\
\text { des G-BA auf Handlungsempfehlungen } \\
\text { für die Erweiterten Landesausschüsse }\end{array}$ \\
\hline $\begin{array}{l}\text { Schnittstellen zu } \\
\text { anderen } \\
\text { Versorgungsberei- } \\
\text { chen }\end{array}$ & & $\begin{array}{l}\text { Wie können Leistungsüberschneidungen } \\
\text { an den Schnittstellen zu anderen Versor- } \\
\text { gungsbereichen verhindert werden? } \\
\text { Schließt eine ASV-Behandlung die } \\
\text { Teilnahme des Patienten an anderen } \\
\text { Versorgungsverträgen aus? }\end{array}$ & $\begin{array}{l}\text { Berücksichtigung der Schnittstellen } \\
\text { zu anderen Versorgungsbereichen bei } \\
\text { Festlegung der ASV-Leistungen } \\
\text { Sicherstellung einer transparenten und } \\
\text { verständlichen Patienteninformation zu } \\
\text { verschiedenen Versorgungsformen }\end{array}$ \\
\hline
\end{tabular}


regelungen bilden dies aber nicht $\mathrm{ab}$ (vgl. Laschet 2011, S. 5; Jaeckel 2012c, S. 6). Bei den niedergelassenen Ärzten erfolgt die Vergütung zwar extrabudgetär, jedoch ziehbaren Strukturen verbleiben, anstatt die neuen Möglichkeiten zu nutzen.

Andererseits erschwert die Komplexität der Lösung auch die Kommunikation mit den Patienten. Wenn sie nicht überzeugt werden können, dass die ASV für sie zu einer spürbar besseren Versorgung führt, droht die Nachfrage nach dieser neuen Versorgungsstruktur sang- und klanglos im Paragra-

zieht sie eine $\mathrm{KV}$-spezifische Budgetbereinigung nach sich, um eine Doppelfinanzierung zu vermeiden. Krankenhäuser mit staatlichen Investitionszuschüssen müssen dagegen einen fünfprozentigen Abschlag akzeptieren. Der Wettbewerb, der durch die Politik ja durchaus gewollt ist, steht somit nicht unter gleichen Vorzeichen.

Eine Wettbewerbsverzerrung wird von der Deutschen Krankenhausgesellschaft auch in Bezug auf die sogenannte Öffnungsklausel bemängelt (vgl. Paquet 2013, S. 30). So kann ein niedergelassener Facharzt je nach Fall entscheiden, ob er im Rahmen der ASV oder der Regelversorgung behandelt, während das Krankenhaus einen potenziellen ASVPatienten nur dann behandeln darf, wenn er die Zugangsvoraussetzungen zur ASV erfüllt. Würde man hier konsequent auf Gleichbehandlung von Krankenhäusern und Niedergelassenen setzen und Krankenhäusern das Recht einräumen, auch Nicht-ASV-Patienten ambulant zu behandeln, müsste man dem niedergelassenen Arzt umgekehrt zugestehen, Patienten stationär zu versorgen. Das kann allerdings kaum gewollt sein.

Das Beispiel zeigt nicht zuletzt, wie schnell die Grenzen der politischen Steuerungsfähigkeit in einem extrem komplexen Umfeld wie dem Gesundheitswesen erreicht sind. Wenn ein Gesetz erst einer jahrelangen Verwaltungsprozedur beim G-BA unterzogen werden muss, bevor es überhaupt zur Anwendung kommen kann, wird die Anfälligkeit dieses Implementierungsansatzes deutlich.

Auf der Strecke bleibt dabei einerseits der klar ersichtliche Nutzen für die Leistungserbringer, die schließlich diejenigen sind, die die neue Versorgungsform am Ende praktizieren sollen. Wenn ein Arzt erst eine mehrtägige Fortbildung absolvieren muss, um Vertragskonstrukte, Vergütungsformen, Rechte und Pflichten zu verstehen, während die finanziellen Anreize relativ klein ausfallen, wird er eher in seinen bewährten und nachvoll- fendschungel unterzugehen.

Die Qualität eines Gesundheitswesens drückt sich auch darin aus, wie schnell es gelingt, innovative Strukturen und Prozesse in die Versorgungspraxis zu implementieren und damit auch positiven Einfluss auf künftige Behandlungsergebnisse zu nehmen. Das Beispiel ASV zeigt noch deutliche Optimierungspotenziale auf, die es in Zukunft zu heben gilt.

\section{Literatur}

Amelung, V.; Wolf, S. (2013) Innovationsförderung in der GKV. In: Gesundheits- und Sozialpolitik, 2-3/2013, S. 111-117

Gemeinsamer Bundesausschuss (G-BA) (2013) Richtlinie ambulante spezialfachärztliche Versorgung § 116 b SGB V (ASV-RL). www.g-ba. de/informationen/beschluesse/1706/

Gesetzestext zur ambulanten spezialfachärztlichen Versorgung (2012) § 116 b SGB V. www.gesetze-im-internet.de/sgb_5/__116b. html

Jaeckel, R. (2012a) Ambulante spezialfachärztliche Versorgung aus der Sicht der Industrie: Vom Drei- zum Vier-Säulen-Modell. In: Market Access \& Health Policy, 01/2012

Jaeckel, R. (2012b) Ambulante spezialfachärztliche Versorgung - Strukturinnovation oder Schreckgespenst für die GKV? In: Welt der Krankenversicherung, 4/2012, S. 112-117

Jaeckel, R. (2012C) Konfliktfeld ambulante spezialfachärztliche Versorgung - Ein neuer Masterplan zur Überwindung der Sektorengrenzen? In: ImpliconPlus - Gesundheitspolitische Analysen, 02/2012, S. 1-10

Laschet, H. (2011) Eine Ordnung für die Spezialmedizin. In: Implicon - Gesundheitspolitische Analysen, 06/2011, S. 1-9

Munte, A. (2013) Die Einführung der Ambulanten Spezialfachärztlichen Versorgung (ASV) nach Paragraph 116 b SGB V. In: ImpliconPlus - Gesundheitspolitische Analysen, 02/2013, S. 1-12

Paquet, R. (2013) Ambulant-spezialfachärztliche Versorgung (ASV): Aus zwei mach eins. In: kma, 5/2013, 18. Jg., S. 28-30

Sachverständigenrat zur Begutachtung der Entwicklung im Gesundheitswesen (SVR)

(2012) Sondergutachten 2012 - Wettbewerb an der Schnittstelle zwischen ambulanter und stationärer Gesundheitsversorgung, Verlag Hans Huber Hogrefe

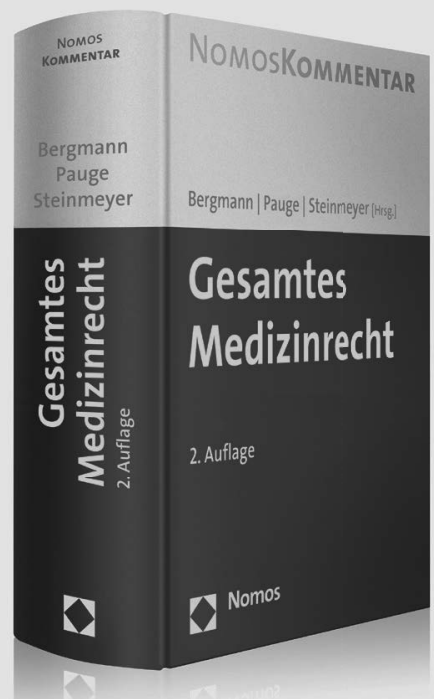

Gesamtes Medizinrecht Herausgegeben von Karl Otto Bergmann, Burkhard Pauge und Heinz-Dietrich Steinmeyer 2. Auflage 2014, ca. 1.800 S., geb., 198,-€

ISBN 978-3-8487-0116-2

Erscheint ca. März 2014

Die Neuauflage schafft zu einem frühen Zeitpunkt Orientierung und Rechtssicherheit. Die Kommentierungen arbeiten die Problemlagen heraus, die die gesetzlichen Neuregelungen in wie der Gesundheitsverwaltung mit sich bringen.

Erstmals kommentiert sind das Embryonenschutzgesetz, die Mediation sowie die Musterberufsordnung für Ärzte. Auch die geplanten Neuregelungen zur Bestechlichkeit von Ärztinnen und Ärzten sind bereits berücksichtigt.

"sollte sich auch in den Bücherregalen sämtlicher Krankenhäuser und Kliniken wiederfinden." G.H. Schlund, Chirurgische Praxis 4/12, zur Vorauflage

www.nomos-shop.de/20247

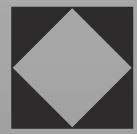

